livraisons

d'Histoire

de l'Architecture

\section{Livraisons de l'histoire de l'architecture}

$18 \mid 2009$

D'architectures

\title{
Exemples d'approche des plafonds à estrich de Moselle orientale
}

Examples to the approach to estrich ceilings in Eastern-Moselle

Untersuchung der Estrichdecken im französischen Ostmoselland

\section{Alexandre Burtard}

\section{OpenEdition}

\section{Journals}

Édition électronique

URL : http://journals.openedition.org//ha/223

DOI : $10.4000 /$ lha. 223

ISSN : 1960-5994

Éditeur

Association Livraisons d'histoire de l'architecture - LHA

Édition imprimée

Date de publication : 10 décembre 2009

Pagination : 51-64

ISSN : 1627-4970

Référence électronique

Alexandre Burtard, «Exemples d'approche des plafonds à estrich de Moselle orientale », Livraisons de I'histoire de l'architecture [En ligne], 18 | 2009, mis en ligne le 10 décembre 2011, consulté le 20 avril 2019. URL : http://journals.openedition.org//ha/223 ; DOI : 10.4000/lha.223

Ce document a été généré automatiquement le 20 avril 2019.

Tous droits réservés à l'Association LHA 


\title{
Exemples d'approche des plafonds à e strich de Moselle orientale
}

\author{
Examples to the approach to estrich ceilings in Eastern-Moselle \\ Untersuchung der Estrichdecken im französischen Ostmoselland
}

\section{Alexandre Burtard}

1 L'architecture et le décor en milieu rural sont aujourd'hui devenus, après des décennies de négligence, des sujets de recherche de premier plan en Lorraine et, plus particulièrement dans la partie orientale du département de la Moselle, où ce patrimoine est encore trop souvent méconnu, donc menacé. Les recherches menées dans ce domaine par les services de l'Inventaire ont notamment permis de repérer et d'étudier un ensemble conséquent de maisons à pan de bois réparties dans soixante-dix communes de l'Est mosellan ${ }^{1}$. De manière succincte, les travaux sur le pan de bois ont mis en évidence quelques exemples d'association entre la technique du colombage et celle des plafonds à estrich, objet de cette étude. Mais, comme on peut le voir au travers d'exemples récemment découverts à Hallering ${ }^{2}$, l'estrich a en fait une véritable singularité. Malgré le succès qu'il a connu de la fin du XVII ${ }^{e}$ siècle jusqu'au milieu du XVIII ${ }^{e}$ siècle au moins, il s'agit sans doute du matériau le moins bien documenté de l'architecture rurale mosellane, en dépit du précieux travail de collecte du folkloriste Louis Pinck, qui, le premier, avait su saisir le particularisme local que constitue l'estrich pour la Moselle de tradition germanique ${ }^{3}$.

\section{Un matériau caractéristique de la Moselle francique}

2 L'estrich (terme désignant à la fois la mise en œuvre et le matériau utilisé) consiste en un mortier de plâtre mêlé à des pierres ou des résidus de tamisage du plâtre, coulé dans les entrevous d'une structure de bois formée par des solives portées par des poutres. Le mot estrich $^{4}$ est issu de la langue allemande, ce qui renforce l'hypothèse d'un lien entre cette technique et son aire géographique d'utilisation: la Moselle francique, où les dialectes 
germaniques sont encore parlés (ill. 1). Son étymologie renvoie selon toute vraisemblance au latin astricum (plâtre).

III. 1 : Carte de la répartition de l'estrich dans le département de la Moselle

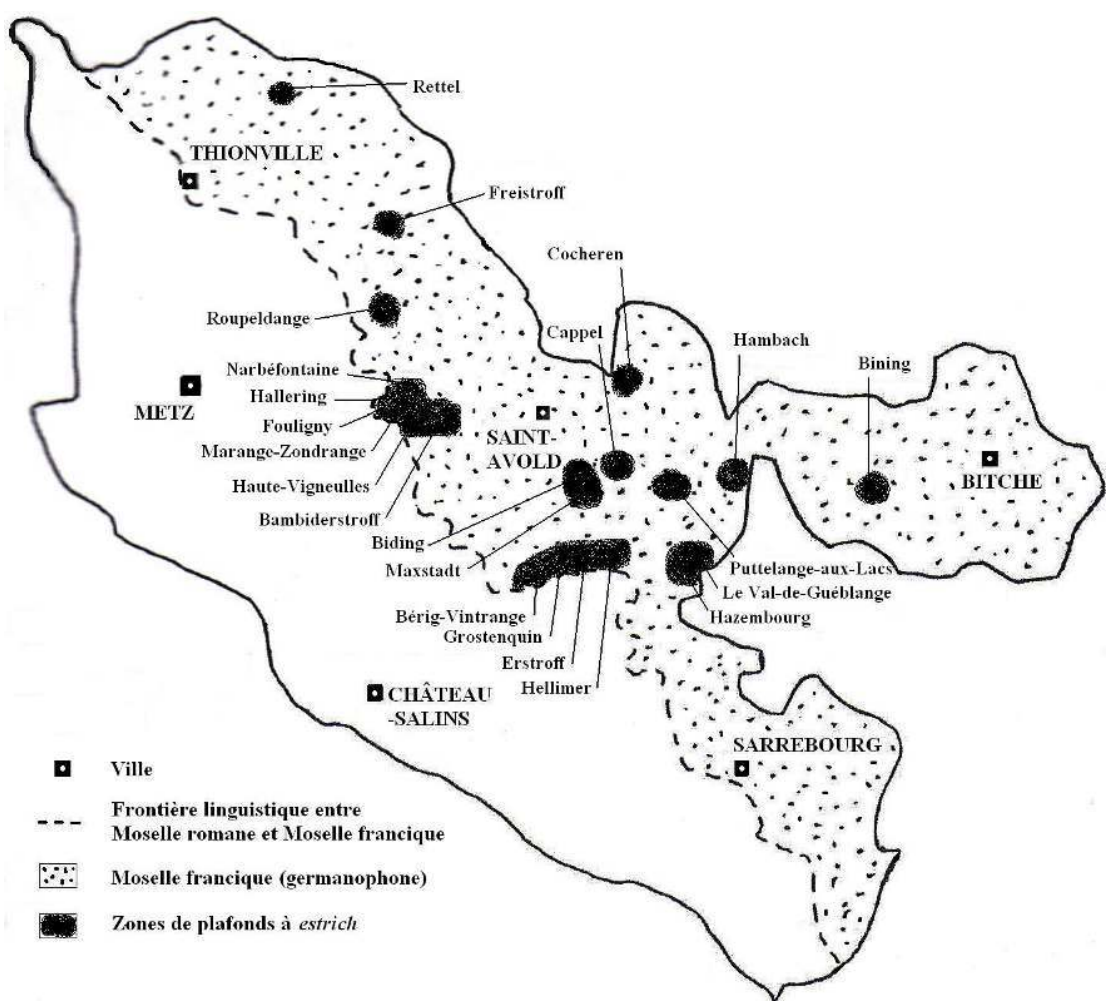

Cl. Alexandre Burtard

3 L'estrich est attesté dès le XVI ${ }^{\mathrm{e}}$ siècle en Lorraine et connaît une utilisation fréquente au moins jusque dans la première moitié du XVIII ${ }^{e}$ siècle, où il s'épanouit avant de disparaître ${ }^{5}$. En Haute-Provence, la même technique a été signalée et étudiée. Elle était en usage au $\mathrm{XV}^{\mathrm{e}}$ siècle ${ }^{6}$. En Lorraine, l'estrich avec motifs moulés semble propre aux espaces germanophones de la Moselle. Á ce jour, il n'a été repéré ni en Alsace ni au Luxembourg mais existe apparemment en Sarre et dans le Palatinat. Cependant, comme dans la langue allemande le mot est encore utilisé quotidiennement pour désigner une chape de ciment, en Allemagne, ou le sol d'un grenier, en Suisse, il n'est pas impossible que ces pays aient connu la technique de l'estrich à une époque ancienne.

4 L'estrich est à la fois un plafonnement et un sol : il n'est pas nécessaire de le recouvrir de plancher ou d'un autre matériau au niveau supérieur ${ }^{7}$ : sa grande résistance permet de le laisser à nu. Dans les maisons encore pourvues d'estrich, on note que le mauvais état éventuel du matériau est davantage dû à des infiltrations d'eau consécutives à l'abandon du bâtiment qu'à une usure naturelle provoquée par près de trois siècles d'occupation. L'eau est en effet un ennemi de l'estrich. Dans un premier temps, elle provoque un assouplissement du plâtre et dégrade les solives de bois, faisant fléchir le plafond sous son propre poids. Dans un second temps, l'estrich perd toute compacité et finit par se déliter.

Outre une grande solidité et des vertus isolantes, l'estrich présente l'intérêt de pouvoir être décoré grâce à des moules en bois, laissant ainsi voir, après décoffrage, des motifs entre les solives du plafond ${ }^{8}$ (ill. 2). 
III 2 : A gauche, moule à estrich provenant de Bambiderstroff, gravé par Jean Muller en 1728. Metz, musées de la Cour d'Or ; à droite, décor de plafond obtenu à partir de ce moule, Hallering.

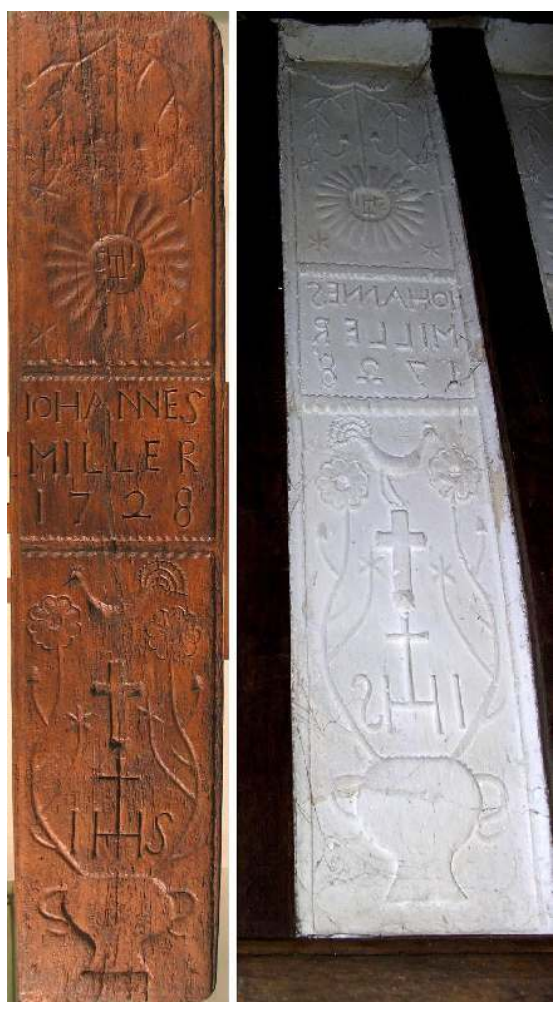

Cl. Alexandre Burtard. Or, il est certain qu'un moule unique servait à réaliser tous les panneaux représentant le même motif 9 . Comme on ne trouve habituellement que deux décors différents au maximum dans une pièce, on imagine aisément les conséquences sur le temps nécessaire au plafonnement d'un seul espace ${ }^{10}$. Cette contrainte de durée est d'autant plus grande si l'on considère la quantité de plâtre à produire puisqu'une dalle d'estrich fait en moyenne quinze centimètres d'épaisseur. Un chantier de plusieurs semaines était vraisemblablement nécessaire pour couvrir l'ensemble d'une maison.

7 Lorsque les entrevous présentent des motifs, on a pris soin de couler, en guise de première couche de la dalle, une pellicule d'estrich vierge de résidus, afin de ne pas nuire ensuite à la finesse du décor. On constate, sur les panneaux où cette fine pellicule est dégradée, qu'elle laisse apparaître un matériau beaucoup plus grossier, dans lequel sont noyées des lattes posées sur les solives, renforçant ainsi la structure et réduisant les risques de fissure de la dalle dans les entrevous. Pour que le décoffrage n'endommage pas le décor en relief, le moule était enduit de savon, d'huile ou de graisse ${ }^{11}$.

Il reste des interrogations quant au coût de la mise en oeuvre, mais l'estrich étant présent aussi bien dans des maisons de modestes journaliers ou artisans que dans des habitats de laboureurs aisés et de notables locaux, le coût ne semble pas avoir été un critère dans son choix comme couvrement.

Dans les quelques villages où le matériau est aujourd'hui connu de la population, c'est principalement en raison de ses décors et les habitants se plaisent à classer les plafonds selon leur beauté et leur état de conservation (ill. 3). 
III. 3 : Vue d'une partie de plafond à estrich représentant des fleurs de lys et des aigles bicéphales

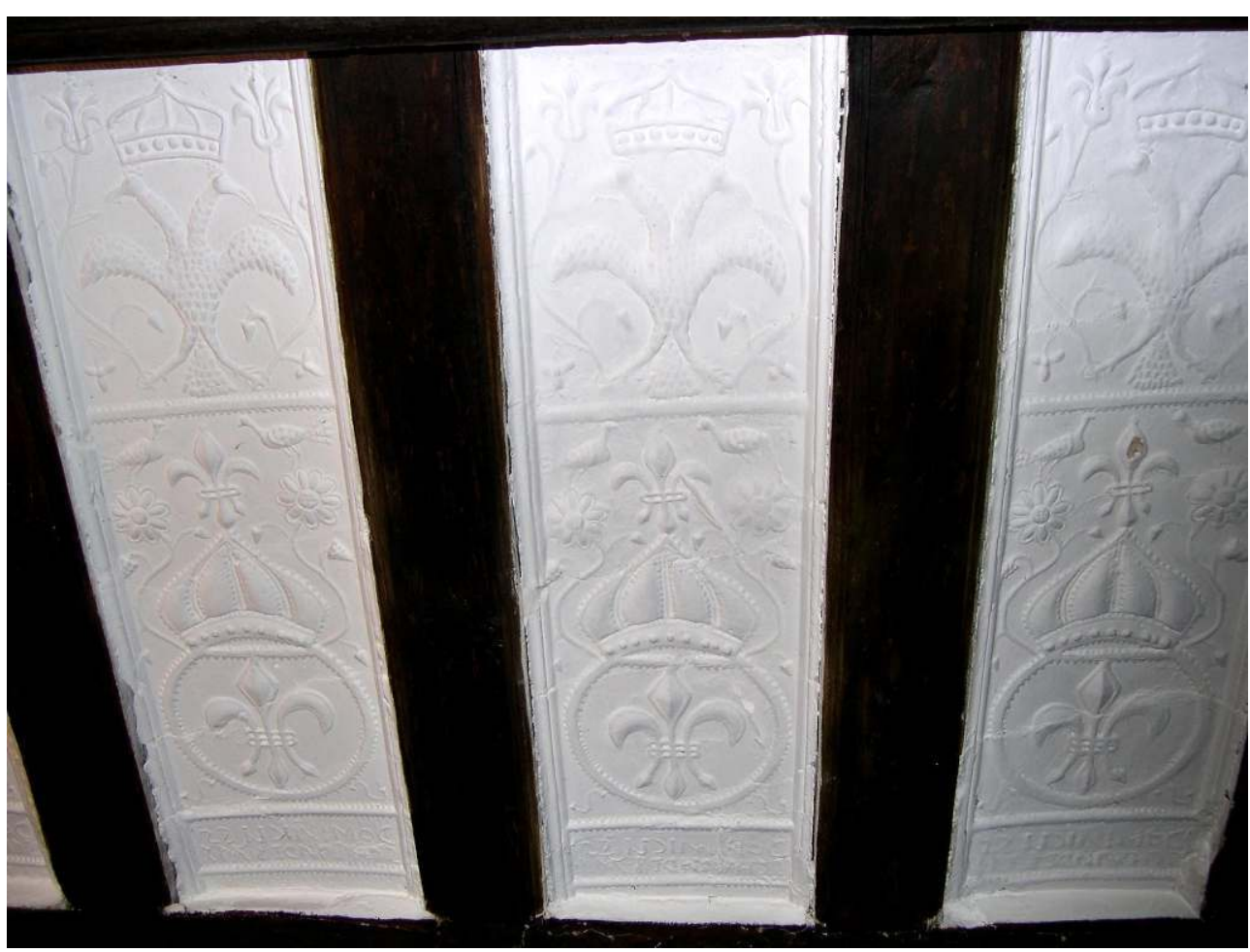

Modèle créé par Dominique Schwind en 1745 à Bambiderstroff, village alors sous souveraineté partagée entre la France et l'Empire

Cl. Alexandre Burtard.

10 Surtout, ces estriche, bien que devenus rares, renseignent le chercheur sur le décor particulier des maisons paysannes de cette micro-région dans la première moitié du XVIII ${ }^{\mathrm{e}}$ siècle et au-delà ${ }^{12}$.

\section{Les maisons à estrich de Hallering}

11 Grâce à une découverte fortuite, nos connaissances sur l'estrich ont pu être approfondies. En effet, la présence d'une ferme entièrement plafonnée en estrich dans la petite commune de Hallering est signalée au service départemental de l'inventaire de Moselle à l'automne 2005. L'importance de la découverte incite la conservatrice à lancer l'inventaire topographique complet de cette commune, d'autant plus qu'une enquête d'urgence avait déjà révélé l'existence de tels plafonds dans la localité en 1991, dans une petite ferme disparue depuis.

12 Sur les cinquante bâtiments formant le noyau ancien du village, six maisons comportant en partie ou en totalité de l'estrich sont attestées ${ }^{13}$. Ce chiffre peut paraître faible, mais la proportion n'est pas négligeable si l'on considère les autres cas répertoriés jusqu'à présent, qui sont souvent des exemples ponctuels ${ }^{14}$. La commune de Hallering se situe en Moselle francique, comme les autres villages où des plafonds à estrich sont connus, à quatre kilomètres seulement de la frontière linguistique séparant le département en deux aires culturelles telle qu'elle se présente aujourd'hui. Hallering ne se trouve pas dans la zone d'expansion du pan de bois, technique à laquelle l'estrich a souvent été associé. 
Toutefois, l'enquête de terrain a montré que le bâti des maisons plafonnées en estrich est fortement marqué par un usage important du bois, que ce soit dans le solivage des plafonds eux-mêmes, dans les encadrements de portes et de fenêtres, ou encore dans le renfort des pignons, des murs de refend et des charpentes au moyen de fermes sur poteaux, grandes ossatures de bois encastrées dans la maçonnerie, technique qui s'apparente au pan de bois.

Trois fermes ou maisons anciennes intéressent particulièrement cette étude, car elles sont datées et comportent toutes trois des panneaux d'estrich avec décor figuré sur leurs plafonds. Ces bâtiments appartiennent à la période comprise entre 1710 et 1750 au cours de laquelle la population dans le duché de Lorraine et dans les enclaves étrangères ${ }^{15}$ augmente fortement. Entre 1712 et 1751, Hallering passe d'environ 40 habitants à 230 . Il est donc logique de trouver dans le bâti actuel du village, les traces de ce développement rapide $^{16}$. Dans la plupart des cas répertoriés, l'estrich est lié à l'architecture de la reconstruction après la guerre de Trente Ans.

La plus petite des maisons à estrich de Hallering est une maison de journalier ou d'artisan ${ }^{17}$. Cette construction, qui ne comprend qu'une travée, porte la date de 1721 sur le linteau de l'entrée. Elle a subi plusieurs dénaturations sur ses façades mais on devine encore les petites fenêtres jumelées à encadrement de bois du début du $\mathrm{XVIII}^{\mathrm{e}}$ siècle, caractéristiques de nombreuses maisons à estrich. Á l'intérieur, la majeure partie des plafonds se compose d'estriche décorés par les motifs annotés $C$ et $D$, le reste étant en panneaux lisses ${ }^{18}$.

15 Le deuxième bâtiment étudié a disparu après l'enquête d'urgence de $1991^{19}$. Il s'agissait d'une ferme de construction homogène dont la porte charretière portait la date de 1720 . Plutôt modeste, elle n'avait d'étage que sur l'avant. Le logis comportait sur certains de ses plafonds deux modèles de panneaux d'estrich, annotés $\mathrm{G}$ et $\mathrm{H}$.

Enfin, il convient d'observer la ferme qui est à l'origine de la découverte ${ }^{20}$. Elle possède le décor de plafond le plus riche, puisqu'on y dénombre pas moins de six modèles de panneaux différents, reprenant les types désignés par les lettres de A à F. Cette ferme, au centre du village de Hallering, remonte, pour ses parties les plus anciennes, aux années 1720. Son plan se divise en deux travées, celle de gauche étant réservée à l'exploitation agricole tandis que celle de droite abrite un logis assez vaste sur deux niveaux ${ }^{21}$. La façade sur rue a été modifiée : l'arc de la porte charretière porte la date de 1806 tandis que certaines ouvertures du logis ont été repercées dans les années 1840.

Ces trois bâtiments permettent d'ores et déjà d'établir plusieurs faits. Tout d'abord, l'utilisation de l'estrich comme matériau de couvrement n'est en rien liée aux moyens financiers des commanditaires, puisque l'architecture des trois édifices montre qu'ils abritaient des propriétaires dont les niveaux de vie étaient différents. L'une des caractéristiques du bâti rural mosellan est en effet de traduire, dans la taille des maisons et les percements présents en façade, à la fois l'activité et la catégorie sociale des occupants d'une maison. L'estrich n'est pas non plus lié à un effet de mode, qui trouverait son origine dans l'habitat aisé pour gagner petit à petit l'habitat modeste: les trois maisons ont été construites autour de 1720 , à des dates très rapprochées, ce qui semble confirmer le statut de l'estrich comme matériau courant, ancré dans la tradition locale. On constate aussi une réutilisation des mêmes panneaux gravés dans des maisons différentes et dans des villages différents. On retrouve par exemple le modèle $G$ sur des plafonds à Hallering, Haute-Vigneulles et Bambiderstroff, localités voisines. Il n'y a donc pas volonté 
de personnaliser le décor d'un habitat, bien que cela ait pu être ponctuellement le cas au moyen de moules particuliers ${ }^{22}$.

18 Les inscriptions et les motifs figurant sur les panneaux permettent d'appréhender la diffusion locale de cet artisanat car la plupart des réalisations de Hallering sont dues à un même artisan, dont la manière, l'évolution et les références iconographiques peuvent être appréciées sur une période de vingt ans.

\section{Des décors et inscriptions d'une rare diversité}

19 Les panneaux gravés ont été signés par leur auteur, ce qui permet aujourd'hui d'attribuer six des huit modèles visibles à Hallering au même artisan. Celui-ci signe tantôt par son nom « Iohannes Miller » (Jean Muller), tantôt par la formule « iohanes handli » (Jean l'a fait) ${ }^{23}$. Malgré cette concentration dans le village de Hallering, l'artisan n'était pas établi dans la localité, les actes paroissiaux de Marange ne mentionnant son nom à aucun moment ${ }^{24}$. Par contre, on trouve à Bambiderstroff un maçon nommé Jean Muller, né vers 1670 et mort en 1751, qui est le seul à pouvoir correspondre à l'artisan recherché25.

Certains estriche ne portent pas la marque de l'auteur ; toutefois, il faut souligner que les modèles sont systématiquement regroupés par paires destinées à un même espace : seul un des panneaux d'une paire est signé, ce qui suffit à l'identification. Dans la grande ferme, les estriche A et $\mathrm{B}$ occupent le couloir, tandis que les autres pièces sont décorées par la paire C-D. Le groupe formé par les panneaux $\mathrm{E}$ et $\mathrm{F}$ est quant à lui visible dans ce qui fut une pièce aménagée au fond de l'écurie ${ }^{26}$. Ce logis est le seul à comporter plusieurs paires dans le village de Hallering; on ne trouve que les modèles $G$ et $H$ dans la petite ferme, et le groupe C-D dans la maison de journalier. L'existence de paires joue un rôle lors de la construction des plafonds. Comme souligné plus haut, l'examen des panneaux montre qu'il n'existe qu'un unique moule pour chaque modèle. Or, le fait de disposer deux panneaux de coffrage différents côte à côte permettait de couler davantage de plâtre en une fois, et réduisait donc de moitié la durée du chantier dans une pièce ${ }^{27}$. C'est pourquoi les plafonds à estrich présentent une alternance aléatoire des modèles d'une même paire, le but étant pour l'artisan de finir le plafond au plus vite, sans se préoccuper de l'harmonie des motifs. De ce fait, il est possible de trouver deux fois de suite un même motif, mais jamais davantage. Il n'est pas non plus tenu compte du sens des panneaux ${ }^{28}$.

21 Au sein d'une paire, l'un des moules porte la date de gravure, indication précieuse pour établir une comparaison avec les dates portées par le bâti. La petite ferme (1720) comprend des plafonds datés de 1728 et 1731. Dans la maison d'ouvrier agricole (1721), on trouve des panneaux de 1719. Quant à la grande ferme (premier quart du XVIII ${ }^{e}$ siècle), les planches de ses estriche ont été gravées en 1708,1719 et 1720 pour les dernières ${ }^{29}$. Ainsi, il n'existe pas de déterminisme entre la construction de la maison et la réalisation des plafonds, d'autant plus que, pour ces derniers, la date portée est celle de la gravure du moule et non celle de la mise en œuvre du matériau. Dans le premier cas, on a attendu plusieurs années avant de couler des dalles en estrich; dans les autres, on a remployé des moules déjà utilisés depuis plusieurs années.

Les motifs moulés par Jean Muller peuvent être regroupés en quatre thèmes développés par l'artisan estrier entre 1708 et 1728, période où il est possible d'observer sa production. Quelques évolutions peuvent être décelées dans ce travail, mais il serait hasardeux d'en tirer des conclusions définitives. En effet, il semblerait que Jean Muller ait gravé une 
paire de moules tous les dix ans, Toutefois, ce rythme de production reste une hypothèse incertaine, car des modèles ont très bien pu disparaître en trois siècles et échapper à notre connaissance ${ }^{30}$.

Les végétaux et les oiseaux constituent le motif récurrent des productions de Muller. Cela semble d'ailleurs être une constante de l'art de l'estrich, puisque la plupart des modèles connus en Moselle sont ornés de branches, de fleurs, de coqs et autres volatiles ${ }^{31}$. Comme dans d'autres foyers de production d'estrich, les tiges des fleurs naissent de vases munis de fines anses. On y trouve roseaux, lys, tulipes, marguerites, roses ou encore pissenlits et fleurs des champs. Quant aux oiseaux, ils sont la plupart du temps posés sur les autres éléments du décor. Le modèle $\mathrm{D}$ présente par exemple un vase contenant une branche accueillant de nombreux oiseaux sur ses ramifications. En 1708 et 1728, Jean Muller adopte le parti de représenter deux bouquets face à face sur ses panneaux gravés, accentuant ainsi l'importance de l'élément végétal. Les bouquets sont conçus selon une symétrie d'ensemble mais pas de détail. En effet, la longueur des tiges de chaque côté est la même, mais les fleurs qu'elles portent peuvent être différentes, rendant ainsi chaque bouquet unique.

III. 4 : A gauche : modèles $A$ et $B$, gravés en 1708 ; à droite : modèles $C$ et $D$, gravés en 1719
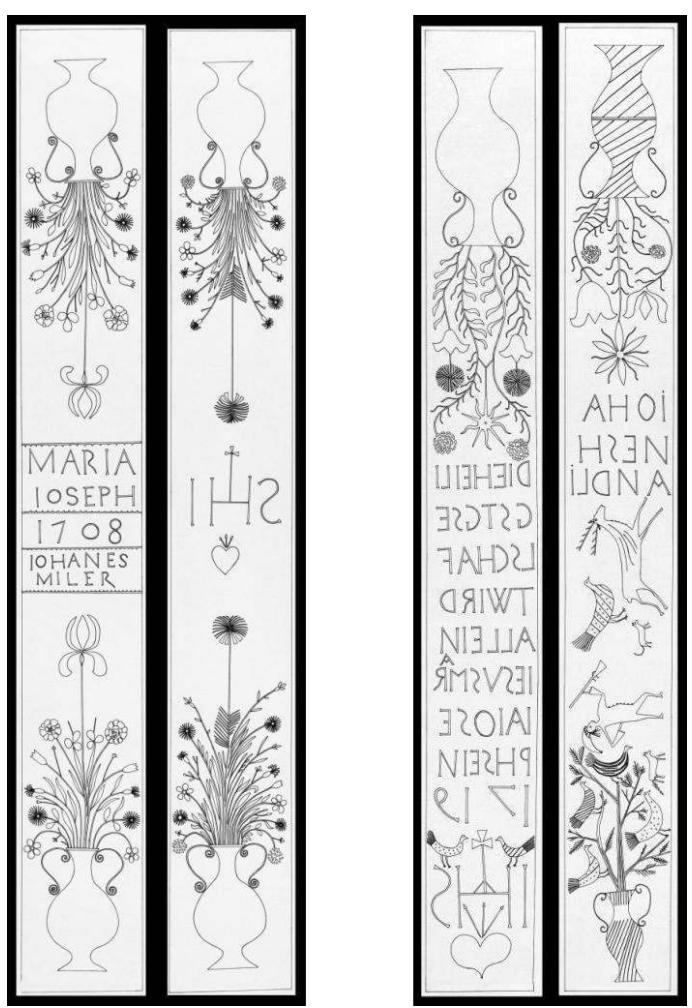

Réalisations de Jean Muller. Relevé : Alexandre Burtard. 
III. 5 : A gauche : modèles $E$ et $F$, gravés en 1720 , réalisations attribuées à Dominique Schwind ; à droite : modèle $G$ (gravé en 1728) et modèle $H$ (gravé en 1731)
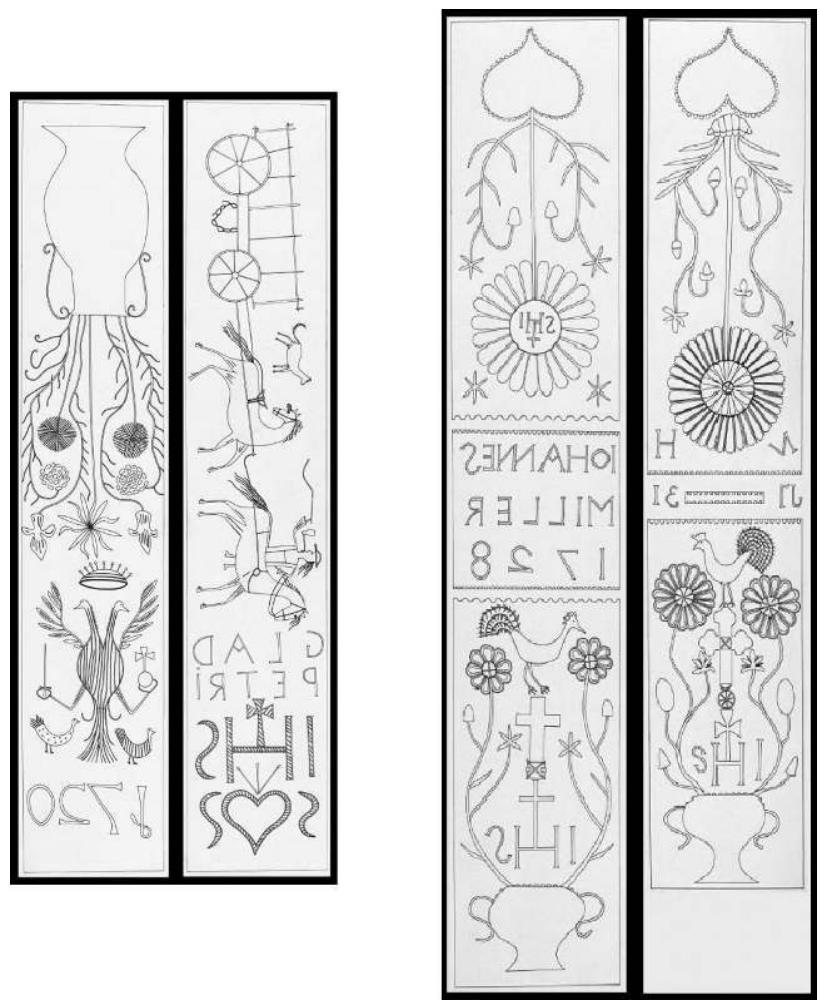

Réalisations de Jean Muller. Relevé : Alexandre Burtard. voit des symboles simples, identifiables par tous : «IHS » crucifères, cœurs surmontés de trois clous, et coqs de saint Pierre. La particularité des plafonds de Hallering est de comporter en outre des inscriptions à caractère religieux: une invocation «Maria Ioseph » gravée en 1708, mais surtout une phrase figurant sur les panneaux de 1719 : « Die heiligst Gselschaft wird allein Iesus Maria Ioseph sein $»^{32}$. On attribuait sans doute à ces sentences, marques d'une piété quotidienne, des vertus de protection à l'attention de la maison et de ses occupants. L'emploi systématique du dialecte traduit l'ancrage local de l'artiste et souligne bien son appartenance et celle du village à la zone germanophone de la Moselle.

L'évolution de Jean Muller, si elle n'est pas notable sur le plan des thèmes choisis, est toutefois indéniable en ce qui concerne le style d'exécution. Tout d'abord, le format adopté pour les moules, très allongé, s'élargit dans ses dernières productions connues ${ }^{33}$. On pourrait en conclure à une amélioration apportée dans la technique ou le matériau entre 1719 et 1728 , permettant de réaliser des entrevous plus larges sans compromettre la solidité des plafonds, mais seule l'utilisation de solives de section plus importante pour porter les modèles plus larges est à signaler.

On note des changements importants dans la manière de l'artiste, principalement entre ses panneaux de 1708 et ses estriche plus tardifs, et il faut bien reconnaître que, sans la signature de l'auteur, il eût été difficile d'y voir le travail du même estrier. Les vases et bouquets de fleurs de 1708 sont très finement dessinés et d'une grande précision, le relief est bas, donnant l'impression d'un décor léger et discret. En revanche, les bouquets 
exécutés en 1719 et 1728 sont davantage stylisés, moins réalistes, les fleurs, en nombre réduit, sont plus grosses. La gravure des moules est plus profonde, le décor est donc nettement plus marqué et sa présence sur le plafond se ressent par le peu d'espaces laissés sans motifs. Quant au schéma d'ensemble, on constate des réminiscences dans le travail de Jean Muller. En effet, la trame des productions de 1708 (deux bouquets opposés séparés par un cartouche à denticules contenant des inscriptions) est reprise en 1728 et 1731 alors qu'elle avait été abandonnée en 1719. Cependant, ce retour n'implique pas une remise en question du style d'exécution adopté dès les panneaux $\mathrm{C}$ et $\mathrm{D}:$ au contraire, le travail sur la représentation des bouquets est encore davantage marqué par un faible souci de réalisme, l'aspect symbolique des dessins étant, semble-t-il, privilégié.

La grande originalité des estriche dans le village de Hallering est de présenter des scènes historiées pour lesquelles on ne connaît actuellement pas d'équivalent dans la zone d'expansion de l'estrich. Le modèle D comporte une chasse au cerf. L'animal est poursuivi par un homme armé, accompagné de son chien, portant un curieux costume qui est apparemment celui d'un rabatteur.

Cependant, le groupe le plus intéressant est sans doute celui formé par les panneaux E et $\mathrm{F}$, commandés en 1720 par le propriétaire de la grande ferme à l'artisan Dominique Schwind. En effet, le modèle F présente l'inscription dialectale « Glad Petri », c'est-à-dire Claude Petry, nom du commanditaire. Cette dédicace est surmontée d'une très belle scène rurale : un homme, fumant une pipe, chevauche en tête d'un attelage et fouette le second cheval qui tire une lourde charrette. Ces évocations sont corroborées par les sources écrites, puisque Claude Petry apparaît régulièrement dans les registres de la paroisse de Marange comme laboureur à Hallering ${ }^{34}$. Son père, Nicolas Petry, avait été maire de justice de Hallering et avait, semble-t-il, bénéficié d'un prestige local important au cours de sa vi ${ }^{35}$. Le motif $\mathrm{F}$ illustre donc parfaitement l'activité professionnelle de l'occupant de la maison. Le fait de commander des moules réalisés sur mesure et gravés à son intention, est, cette fois, une marque de l'aisance financière du propriétaire, car ils ne pourront pas être réutilisés. Le panneau $\mathrm{E}$, associé au précédent, renforce le prestige, le rôle politique et l'ancrage local de la famille Petry. En effet, on y voit une aigle impériale bicéphale, surmontée d'une couronne, tenant dans ses serres un globe et une épée, symbole éminemment évocateur. Il s'agit, une fois de plus, d'une référence à l'espace germanique qui influence fortement cette région dont l'estrich pourrait constituer un particularisme architectural et ornemental ${ }^{36}$.

Les estriche halleringeois représentent un apport non négligeable à notre connaissance du décor des maisons en milieu rural : ils sont riches d'enseignement sur ce matériau peu étudié, qui a pourtant connu un développement important entre les années 1690 et 1760 en Moselle orientale, sans que les raisons exactes de la diminution de son utilisation ne soient clairement établies ${ }^{37}$. La diversité des motifs présents dans le village de Hallering dévoile quantité d'informations jusqu'alors insoupçonnées en matière de décor moulé de plafond en Moselle et montre qu'il existait une véritable attention portée à l'ornementation intérieure des maisons.

Surtout, c'est le travail de deux artisans en particulier qu'il est permis de suivre sur une vingtaine d'années. On peut apprécier à quel point ce travail d'art populaire, marqué par la répétition, témoigne en fait de la grande créativité des artisans du XVIII ${ }^{e}$ siècle, à la fois maitres d'œuvre et décorateurs. En fait de naïveté, il faut plutôt y voir une habileté à retranscrire dans le plâtre un foisonnement de symboles. L'estrich, en tant que support 
iconographique et écrit, montre à quel point la société rurale était pénétrée de références culturelles dont l'étude révèle aujourd'hui la diversité et la profondeur.

\section{NOTES}

1. Enquêtes et travaux notamment menés par Jacques Guillaume et Jean-Yves Chauvet pour le service régional de l'Inventaire de Lorraine.

2. . Les communes citées sont toutes de petite taille et en milieu rural. Elles sont situées aux confins du pays messin, du bassin houiller lorrain et du pays du Saulnois. Cette étude, qui expose principalement les découvertes récentes sur le matériau, s'inscrit dans le cadre d'un travail de plus grande ampleur effectué pour le service départemental de l'Inventaire et du Patrimoine de la Moselle en 2006 dans le but de recenser et d'étudier tous les modèles d'estrich connus dans ce département. Le fruit de cette recherche a donné lieu à la rédaction d'un mémoire de Master 2 soutenu la même année par l'auteur à l'université de Nancy 2 sous la direction du professeur Philippe Martin.

3. L'abbé Louis Pinck (1873-1940) fut l'un des grands défenseurs de la culture et de la langue germaniques de la Moselle orientale. Il a notamment publié en quatre tomes, entre 1926 et 1939, le fruit d'un travail ethnolinguistique conséquent, intitulé Verklingende Weisen. Cet ouvrage est un recueil de plus de 2000 chansons populaires (paroles et musique) de Moselle germanophone collectées auprès de plusieurs centaines de témoins de 1913 à 1938. La collection Pinck contenait également des meubles traditionnels (dispersés) et un important fonds photographique, conservé aux archives municipales de Sarreguemines. C'est dans ce fonds que se trouvent des relevés de décors d'estrich de la région de Puttelange-aux-Lacs. Ces relevés ont été effectués par l'architecte Koenig pour le compte de Pinck en 1937 et se révèlent aujourd'hui d'une grande valeur dans l'étude menée sur l'estrich.

4. . Le mot étant d'origine allemande, il prend un « $\mathrm{e}$ » lorsqu'il est au pluriel.

5. . Des motifs du XV $\mathrm{XV}^{\mathrm{e}}$ siècle, réalisés selon une technique légèrement différente, existent dans la maison de la Dîme de Rettel. Les originaux de ces modèles ont été transférés en 1905 au château du Haut-Koenigsbourg (Bas-Rhin). En effet, dans une optique folkloriste, une chambre lorraine a été aménagée dans le logis lors de la restauration de ce nouveau palais wilhelminien; une manière de symboliser la pleine appartenance de la Moselle à l'Alsace-Lorraine annexée par l'Empire allemand. Le fait d'avoir alors considéré l'estrich comme représentatif de cette partie de la province est en soi très intéressant.

6. . Élizabeth Sauzé, "L'Art de la gypserie à Riez au XV e siècle ", dans Provence historique, fasc. 167-168, Aix-en-Provence, 1992, p. 297-310.

7. . Ce parti a toutefois été adopté dans certains cas, dès l'origine ou encore au XIX ${ }^{\mathrm{e}}$ siècle, en même temps que l'on cachait les décors par des faux plafonds en lambris.

8. . Le musée de Metz possède dans ses collections une planche de coffrage gravée, qui semble être, à ce jour, la seule conservée. Son motif est également le plus répandu dans le village de Bambiderstroff, d'où elle provient, et dans les environs. Elle est entrée au musée en 1924 et a alors fait l'objet d'un compte-rendu devant la société d'histoire et d'archéologie de Lorraine. Voir Émile Linckenheld, "un moule de plafonneur lorrain en 1728 ", dans Cahiers lorrains, t.VI, juin 1924, p. 82-83.

9. . Les défauts éventuels se retrouvent en effet sur tous les exemplaires d'un même motif. 
10. . Toutefois, la prise du matériau devait être rapide (même si le séchage pouvait prendre plus de temps, comme pour un plâtre traditionnel), permettant ainsi de passer assez vite aux entrevous suivants. Ces remarques concernant la durée du chantier ne s'appliquent pas aux plafonds non décorés, pour lesquels le coffrage, en panneaux lisses, pouvait être mis en place entièrement avant de couler le matériau. Paradoxalement, ces plafonds à estrich lisse sont plus rares.

11. . Abbé Louis Pinck, Verklingende Weisen, tome III, Kassel, 1963, p. 168 et notes p. 477-478. (édition originale en quatre tomes, s.l., 1926-1939).

12. . Le milieu urbain ne semble en effet pas touché par l'utilisation de l'estrich. La première moitié (et plus particulièrement le premier quart) du XVIII ${ }^{\mathrm{e}}$ siècle est la période pour laquelle le plus grand nombre de panneaux d'estrich à motifs sont encore conservés. Mais il faut souligner que ces moules étaient encore utilisés bien après leur date de gravure, il est donc difficile de dater précisément certaines réalisations.

13. De l'estrich est conservé dans quatre de ces bâtiments, en ce qui concerne les deux autres, l'un a été récemment transformé entraînant la disparition de panneaux lisses, l'autre a été entièrement démoli en 1991 après inventaire. De plus, certaines maisons sont suspectées d'avoir eu par le passé des plafonds à estrich. Les personnes âgées interrogées indiquent en effet que de tels plafonds ont pu disparaître dans la première moitié du $\mathrm{XX}^{\mathrm{e}}$ siècle au profit de planchers traditionnels en bois.

14. . Un village voisin, Bambiderstroff, possède une concentration beaucoup plus élevée de plafonds en estrich. L'enquête menée parallèlement à Hallering et Bambiderstroff révèle que cette seconde localité fut le foyer de l'estrich dans ce secteur. Il y existe beaucoup de modèles différents, signés par plusieurs artisans qui résidaient dans ce village, comme le confirment les registres paroissiaux.

15. . Hallering est à ce moment sous la souveraineté du Luxembourg, entouré par des terres lorraines et françaises.

16. . Alexandre Burtard, Les Mémoires de nos trois clochers, Marange-Zondrange-Hallering, 2004, $430 \mathrm{p}$.

17. . Au $\mathrm{n}^{\circ} 30$, rue Principale.

18. . Les illustrations 4 et 5 présentent les relevés des huit motifs cotés de $\mathrm{A}$ à $\mathrm{H}$, présents dans les maisons de Hallering.

19. . Au n ${ }^{\circ} 27$, rue Principale.

20. . Au n 39 a, rue Principale.

21. En Moselle, une travée d'habitation comporte généralement, dans le bâti rural traditionnel, trois pièces en enfilade, celle du centre, aveugle, étant la cuisine dont une moitié est occupée par un âtre. La plupart des logis sont également dotés de trois pièces en enfilade à l'étage.

22. . La grande ferme de Hallering est un exemple de cette pratique, comme cela est expliqué plus bas.

23. . Toutes les inscriptions sont en dialecte francique, elles sont restituées dans l'orthographe figurant sur les estriche. Notons que les panneaux E et F ne sont pas signés; il est toutefois fort peu probable qu'ils soient dus à un second auteur resté anonyme au vu de leur parenté stylistique avec les panneaux signés.

24. . Jean-Marie Pira, Reconstitution des familles 1680-1900: Hallering, 2001, 109 p.; et MarangeZondrange, Cercle généalogique du Pays naborien, 2003, 210 p. Précisons que Hallering est, sur le plan religieux, une annexe de la paroisse de Marange.

25. . De plus, rappelons que le moule gravé conservé au musée de Metz et portant la signature de Jean Muller provient de Bambiderstroff. Dans ce village, plusieurs maisons (au moins une dizaine) conservent encore leurs plafonds anciens, sans compter les bâtiments disparus qui étaient connus pour contenir de l'estrich. 
26. . Le groupe E-F est vraisemblablement dû à l'estrier Dominique Schwind, de Bambiderstroff ; c'est du moins ce que laisse penser la présence sur le panneau $\mathrm{F}$ d'un double $\mathrm{S}$ repéré également sur un panneau signé de cet artisan à Bambiderstroff.

27. Curieusement, on n'a pas cherché à associer plus de deux moules pour un même plafond. Il faut peut-être y voir une limite dans le rendement de la production de plâtre. Dans l'hypothèse d'une production faible et lente, plus de deux moules auraient été inutiles.

28. Ce n'est pas le cas à Bambiderstroff où une attention plus grande a été portée à ces détails ayant leur importance dans l'aspect final du décor.

29. . Dates gravées sur les estriche de Hallering : paire A-B : 1708 ; paire C-D : 1719 ; paire E-F : 1720 ; modèle $G: 1728$; modèle $H: 1731$.

30. . C'est d'ailleurs ce que l'étude de terrain menée à Bambiderstroff laisse entrevoir puisque davantage de modèles différents, également datés et parfois signés de Jean Muller ou d'autres artisans, sont conservés dans cette localité.

31. . Le monde végétal et les symboles religieux dans les décors d'estrich reçoivent le même traitement que dans l'ébénisterie lorraine traditionnelle, où ils sont des motifs récurrents. Cette transposition des thèmes se retrouve également dans la faïence et la broderie.

32. Cette phrase en dialecte signifie : « La plus sainte compagnie sera uniquement celle de Jésus, Marie et Joseph ». Á ce jour, le panneau C est le seul connu pour comporter une phrase entière et non pas de simples noms. Comme pour toutes les inscriptions figurant sur les estriche de Hallering (à une notable exception pour le panneau A), le texte a été gravé à l'endroit sur le moule et figure donc à l'envers sur le plafond. C'est là l'une des limites du savoir-faire des estriers en matière de gravure.

33. . Ce format est d'environ 160 par 22 centimètres en 1708 et 1719 , il passe à 180 par 37 centimètres en 1728.

34. . Claude Petry est né en 1681 et mort en 1751 à Hallering. Lui et son épouse, ainsi que ses parents et deux de ses enfants restés à Hallering sont tous inhumés à l'intérieur de l'église paroissiale de Marange.

35. . Nicolas Petry, mort en 1703, avait dû se réfugier en 1677 dans le bourg lorrain de Longeville, non loin, où il bénéficiait certainement de la protection de l'abbé de Saint-Martin-des-Glandières pendant l'occupation des terres lorraines et des enclaves luxembourgeoises par la France. C'est en tout cas ce que suggèrent les indications portées dans les registres des actes religieux de Marange-Hallering. Jean-Marie Pira, op. cit.

36. Cette référence est d'autant plus intéressante que le Luxembourg, dont dépendait Hallering, venait de passer en 1715 , soit cinq ans avant la gravure du moule E, de la domination des PaysBas espagnols à celle de l'Empire d'Autriche.

37. Si l'utilisation abondante de l'estrich se situe entre 1690 et 1760 , la technique n'a toutefois pas disparu de manière brutale, les moules ont été utilisés encore longtemps après avoir été gravés, bien qu'il soit difficile de préciser jusqu'à quelle époque. Á partir du XIX ${ }^{\mathrm{e}}$ siècle, la mise en œuvre d'estrich dut être très ponctuelle, mais un témoignage recueilli à Bambiderstroff atteste la construction d'un plafond en estrich, disparu, dans cette localité à la veille de la Première Guerre mondiale. 


\section{RÉSUMÉS}

Les plafonds à estrich sont des décors caractéristiques de la Lorraine germanophone. On les trouve en milieu rural, dans d'anciennes fermes et maisons paysannes des XVIII ${ }^{\mathrm{e}}$ et XIX ${ }^{\mathrm{e}}$ siècles. Il s'agit de plafonds stuqués mis en œuvre grâce à des moules en bois gravés de signes, inscriptions et motifs décoratifs, pouvant ainsi être reproduits à l'envi. Cette technique n'existe en France que dans la micro-région est-mosellane mais se trouve selon toute vraisemblance également en Sarre et dans le Palatinat. En 2006, une découverte fortuite de plafonds à estrich dans une ferme de la commune de Hallering a conduit le service départemental de l'inventaire et du patrimoine de la Moselle à mener un recensement de ces décors si particuliers, dont beaucoup ont déjà disparu ou sont menacés de disparaître, en raison des conditions dans lesquels ils sont conservés, dans des lieux parfois humides, abandonnés ou sujets à des réhabilitations ne tenant pas compte de leur présence. Les plafonds nouvellement découverts à Hallering sont particulièrement intéressants car, outre des inscriptions et des signatures, ils comportent également des scènes historiées et des symboles politiques. Ils permettent aussi de suivre l'évolution de la production particulière de quelques artisans du XVIII ${ }^{\mathrm{e}}$ siècle.

In German-speaking Lorraine are some very particular decoration still preserved in old farms of the countryside. In those houses, built during the eighteenth and the nineteenth centuries, the ceilings are made of plaster moulded on an exposed beams structure. The wooden matrix, that has been carved by a creative craftsman, is used to reproduce the decoration over and over again. This technique is only known in this small territory of Eastern Lorraine but seems to exist in Germany as well (especially in the Saar and the Palatinate). 2006 another estrich ceiling happened to be found in a small village called Hallering. This fact persuaded the curatorial office of Heritage of Moselle to lead an inventory of those decorated ceilings. In fact, a lot of estrich ceilings are endangered: an important part has already disappeared during the twentieth century because the gypsum is very fragile when exposed to water in abandoned houses. The estrich ceilings are very interesting because the decorated face bear not only dedications and signatures but historiated scenes and political signs too. Therefore it is possible to study the works of a small circle of craftsmen decorating rural housings in the eighteenth century.

Im deutschsprachigen Lothringen sind in alten Bauernhäusern aus dem 18. oder 19. Jahrhundert noch ganz besondere Ornamente vorhanden, so zum Beispiel in Holzgießformen abgedruckte Stuck-Gipsdecken, deren Muster von kreativen Handwerkern eingraviert wurden. Diese Technik hat sich in Frankreich nur in diesem begrenzten Gebiet entwickelt, aber sie hat anscheinend auch in Deutschland existiert, und zwar im Saarland und in der Pfalz. Nach einer neuen Entdeckung von Estrichdecken 2006 in einem alten Bauernhaus in der Gemeinde Halleringen sah sich die Kulturbehörde des Departement Mosel verpflichtet, eine Inventar von diesen Ornamenten anzulegen.Es ergibt sich, dass die Stuck-Gipsdecken heute in Ostlothringen ausgesprochen gefährdet sind. Da dieses weiche Material die Feuchtigkeit der verlassenen Häuser schlecht aushält, sind sogar davon viele schon verschwunden. An den besonders interessanten neuentdeckten Putzdecken lassen sich nicht nur In- und Unterschriften erkennen, sondern auch ländliche Szenen sowie politische Zeichen. Es wird also die Fertigkeit der kleinen 
Handwerkerkreise vor Augen geführt, die im 18. Jahrhundert zu der Verzierung der Bauernhäuser beigetragen haben.

\section{AUTEUR}

\section{ALEXANDRE BURTARD}

Alexandre Burtard est né en 1982. Il effectue une hypokhâgne à Metz et une khâgne à Reims, avant de suivre des études à l'université de Nancy 2. Il se spécialise en histoire et obtient en 2004 une maîtrise sous la direction du professeur Philippe Martin. Son mémoire, intitulé Les paroisses de l'archiprêtré de Varize et la Réforme tridentine lui permet d'étudier la pénétration des prescriptions du concile de Trente dans une partie de la Lorraine, notamment en ce qui concerne le découpage administratif, l'architecture et le mobilier liturgique. En 2006 il soutient, sous la direction des professeurs Philippe Martin et Pierre Sesmat, un mémoire de master 2 intitulé Plafonds à estrich de Moselle orientale dans le cadre de l'Inventaire du patrimoine (XVII ${ }^{e}$ siècle - XIX ${ }^{e}$ siècle), qui lui vaut l'année suivante le prix d'histoire de l'académie nationale de Metz. Il effectue alors, sous la direction de Marie Gloc, conservateur au service de l'inventaire et du patrimoine de la Moselle, un travail de recensement et d'étude des décors de plafond en milieu rural caractéristiques de la Lorraine germanophone. Phillipe Martin lui donne également l'occasion de collaborer à l'ouvrage collectif Metz, 2000 ans d'histoire, paru aux éditions Serpenoise en 2007. Après deux années en classe préparatoire au concours de conservateur à l'École du Louvre, Alexandre Burtard effectue plusieurs stages, notamment au musée d'Orsay où il achève l'inventaire du fonds du peintre-décorateur Charles Lameire (1832-1910) et poursuit le travail de documentation sur l'artiste, et à la conservation des œuvres d'art religieuses et civiles de la Ville de Paris. Également conférencier national et guide-interprète, Alexandre Burtard est actuellement chargé d'études à la direction de l'urbanisme de la Ville de Paris, dans le cadre de la révision du plan de sauvegarde et de mise en valeur du quartier du Marais. Adresse électronique : alexandre.burtard@hotmail.fr 